\title{
Integrin A6 Cleavage in Mouse Skin Tumors
}

\author{
Manolis C. Demetriou ${ }^{1}$, Kevin A. Kwei ${ }^{2}$, Marianne B. Powell ${ }^{2}$, Raymond B. Nagle ${ }^{3}$, G. Tim Bow- \\ $\operatorname{den}^{4}$, and Anne E. Cress*,4
}

\begin{abstract}
From the ${ }^{1}$ Department of Biological Sciences, University of Cyprus, Cyprus, the ${ }^{2}$ Department of Radiation Oncology, Stanford University, Stanford, California and the ${ }^{3}$ Department of Pathology, and the ${ }^{4}$ Department of Cell Biology and Anatomy, University of Arizona, Tucson, Arizona, USA
\end{abstract}

\begin{abstract}
We have previously identified a structural variant of the $\alpha 6$ integrin (Laminin receptor) called $\alpha 6 \mathrm{p}$. The $\alpha 6 \mathrm{p}$ variant is a $70 \mathrm{kDa}$ form of the full-length $\alpha 6$ integrin $(140 \mathrm{kDa})$ that remains paired with either the $\beta 1$ or $\beta 4$ subunit on the cell surface. $\alpha 6 \mathrm{p}$ is produced by urokinase-type plasminogen activator (uPA), which removes the extracellular $\beta$-barrel domain while the receptor is on the cell surface. The $\alpha 6 \mathrm{p}$ integrin was present in human prostate cancer tissue but not in normal tissue and the cleavage of the $\alpha 6$ integrin extracellular domain promotes tumor cell invasion and migration on laminin. The objective of the present study was to determine whether the $\alpha 6 \mathrm{p}$ integrin is observed in other models of carcinogenesis. Our results indicate detectable low levels of $\alpha 6 \mathrm{p}$ in normal mouse skin, and comparatively elevated levels in mouse papillomas and squamous cell carcinomas induced by DMBA, TPA and MNNG treatments. Furthermore, we have found that $\alpha 6 \mathrm{p}$ was present at high levels in skin melanomas of transgenic mice that over express activated Ha-ras under the control of the tyrosinase promoter. Finally, subcutaneous injection into athymic nude mice of a malignant mouse keratinocyte derived cell line (6M90) that is $\alpha 6 \mathrm{p}$ negative, results in the development of tumors that contain $\alpha 6 \mathrm{p}$ integrin. The latter results indicate that $\alpha 6 \mathrm{p}$ is induced in vivo suggesting that the tumor microenvironment plays a major role in the production of $\alpha 6 \mathrm{p}$. Taken together, these data suggest that the cell surface cleavage of the $\alpha 6$ integrin may be a novel mechanism of integrin regulation and might be an important step during skin tissue remodeling and during carcinogenesis.
\end{abstract}

\section{INTRODUCTION}

Integrins are heterodimeric cell surface proteins composed of $\alpha$ and $\beta$ subunits. Each $\alpha \beta$ combination has its own binding specificity and signaling properties [1,2]. Different integrins recognize different extracellular matrix (ECM) proteins $[1,2]$. They are involved in many processes including cell migration, differentiation, blood clotting, tissue organization and cell growth. Integrins generally contain a large extracellular domain ( $\alpha$ subunit $\sim 1000$ residues, and $\beta$ subunit $\sim 750$ residues), a transmembrane domain and a short cytoplasmic domain ( $\sim 50$ residues or less) with the exception of $\beta 4$, whose cytoplasmic domain is large (more than 1000 residues) [3, 4].

Our previous work has shown that a structural variant of the $\alpha 6$ integrin called $\alpha 6 \mathrm{p}$ exists [5]. This variant is missing the extracellular $\beta$-propeller domain associated with ligand binding [5]. We have also shown that $\alpha 6 \mathrm{p}$ is produced by proteolytic cleavage of the $\alpha 6$ integrin by Urokinase-type Plasminogen Activator (uPA) [6]. The $\alpha 6 \mathrm{p}$ integrin is 70 $\mathrm{kDa}$ protein, and mass spectrometry analysis showed that the $\mathrm{NH}_{2}$ terminal end of the molecule contains at least amino acids starting at arginine 595 [5]. Using a multiple sequence alignment tool, this position in the $\alpha 6$ integrin lies within an accessible loop upstream from the genu region described for the $\alpha \mathrm{V}$ integrin subunit[7]. Using site-directed mutagenesis we have identified residues R594 and R595 as essential for cleavage [8].

\footnotetext{
*Address correspondence to this author at the Department of Cell Biology and Anatomy, University of Arizona, Tucson, Arizona, USA; E-mail: acress@azcc.arizona.edu
}

The protease that cleaves the $\alpha 6$ integrin, uPA, is a secreted 54-kDa serine protease which cleaves plasminogen as a primary substrate [9]. uPA has also been shown to catalyze the proteolytic cleavage of the extracellular matrix protein fibronectin [10], hepatocyte growth factor/scatter factor (HGF/SF) [11] and macrophage-stimulating protein (MSP) [12]. The expression of uPA has been shown to be correlated with many cancers. In addition, TPA treatments have been shown to increase uPA levels in cell lines and mouse skin [6, 13, 14]. Moreover, we have previously shown that TPA induces a uPA dependent $\alpha 6$ integrin cleavage in normal breast cells MCF10A [6]. Also, MNNG treatments have been shown to induce uPA expression in different cell lines [15].

The objective of the present study was to determine if $\alpha 6 p$ integrin was present in other models of carcinogenesis, to examine if the $\alpha 6 \mathrm{p}$ integrin is present in mouse skin tumors, and to determine whether $\alpha 6 \mathrm{p}$ could be induced in vivo. We have analyzed normal mouse skin, skin tumors induced by DMBA, TPA and MNNG treatments, tumors from Ha-ras transgenic mice that were treated with DMBA, and tumors from subcutaneous injection of tumorigenic cell lines into athymic nude mice. Our results showed that $\alpha 6 \mathrm{p}$ is detectable in low levels in nomal mouse skin and its expression is elevated in mouse skin tumors. These data suggest that the cleavage of the $\alpha 6$ integrin occurs in epithelial tissues other than prostate and can be induced in vivo.

\section{MATERIALS AND METHODOLOGY}

\section{Reagents}

The DU145 human prostate cancer cell line was grown in Dulbecco's Modified Eagle's Medium (DMEM) (Gibco BRL: Gaithersburg, MD, USA) plus $10 \% \%$ fetal bovine 
serum (FBS) and incubated at $37^{\circ} \mathrm{C}$ in a humidified atmosphere of $95 \%$ air and $5 \% \mathrm{CO}_{2}$.

The AA6A rabbit polyclonal antibody was raised and purified by Bethyl Laboratories Inc. (Montgomery, TX, USA). AA6A is specific for the last 16 amino acids (CIHAQPSDKERLTSDA) of the human $\alpha 6 \mathrm{~A}$ sequence [17], as described previously [18] and will recognize full-length $\alpha 6$ as well as full-length $\alpha 3$ due to amino acids QPS. Since $\alpha 3$ is not cleavable, $\alpha 6 \mathrm{p}$ can be readily detected at $70 \mathrm{kD}$ on the western blot. DMBA was purchased from Sigma (Sigma, St. Louis, MO), and TPA from Alexis Corp. (Alexis Corp., San Deigo, CA).

\section{Western Blotting}

Protein samples (50ug for tissues or 20ug for cell lines) were diluted in $2 X$ non-reducing sample buffer and the samples were boiled for $5 \mathrm{~min}$, and after a quick chill on ice, they were loaded onto $7.5 \%$ SDS-PAGE. Proteins resolved in the gel were electrotransferred to Millipore Immobilon-P polyvinylidene fluoride (PVDF) membrane (Millipore, Bedford, MA, USA), incubated with primary antibody and after washing with secondary antibody conjugated to horseradish peroxidase and visualized by chemiluminescence (ECL Western Blotting Detection System, Amersham, Arlington Heights, IL, USA).

\section{Mouse Tissue Analyses}

A single initiating dose of $100 \mu \mathrm{g}$ DMBA (Sigma, St. Louis, MO) in $0.2 \mathrm{ml}$ of acetone was applied topically to the shaved backs of female ICR mice (Harlan Sprague Dawley, Indianapolis, IN). It was followed by bi-weekly application of $5 \mathrm{nM}$ TPA (Alexis Corp., San Deigo, CA) in $0.2 \mathrm{ml}$ of acetone for 21 weeks. At week 22, TPA applications were stopped and replaced with weekly application of $120 \mu \mathrm{g}$ MNNG in $0.2 \mathrm{ml}$ of acetone for a total of 40 weeks. For normal tissue, untreated mice were either unshaved and the whole skin was removed and frozen in liquid nitrogen, or they were treated with hair removing cream to remove the hair and the skin was harvested and frozen in liquid nitrogen. The tumors were collected from the treated mice and were frozen in liquid nitrogen. The samples were homogenized by mortar and pestle and lysed in RIPA buffer. The samples were centrifuged at $14000 \mathrm{RPM}$ at $4^{\circ} \mathrm{C}$ for 15 minutes and the supernatants collected and sonicated. The resulting sample (50ug) was analyzed on a 7.5\% SDS-PAGE gel, and a Western blot was performed using the AA6A antibody.

The transgenic mice that express an activated Ha-ras gene in their pigmented cells were described previously [19]. Melanoma tumors in different stages from these mice that were treated with DMBA were isolated and protein was extracted using RIPA lysis buffer. The samples were then analyzed by western blotting for the $\alpha 6$ or $\alpha 3$ and $\alpha 6 p$ integrins using the AA6A rabbit polyclonal antibody.

The 6M90 cells were injected subcutaneously in athymic nude mice and the tumors were isolated and protein was extracted using RIPA lysis buffer. The samples were then analyzed on an SDS-PAGE gel and the $\alpha 6$ or $\alpha 3$ and $\alpha 6 p$ integrins were detected using the AA6A antibody.

\section{RESULTS}

\section{Detection of $\alpha 6 p$ in Normal Mouse Skin, Papillomas and} Squamous Cell Carcinomas

We have previously shown that $\alpha 6 p$ exists in human prostate cancer tissue but not in normal prostate tissue. This prompted us to investigate the $\alpha 6 \mathrm{p}$ expression in other tissues. We first analyzed normal mouse skin isolated from C57BL6 and ICR CD1 mice. We analyzed unshaved skin as a non-treated control and shaved skin that was treated with hair removing cream to remove the hair from mouse backs. Our results in Fig. (1) indicated that normal mouse skin contains low but detectable levels of $\alpha 6 \mathrm{p}$ integrin.

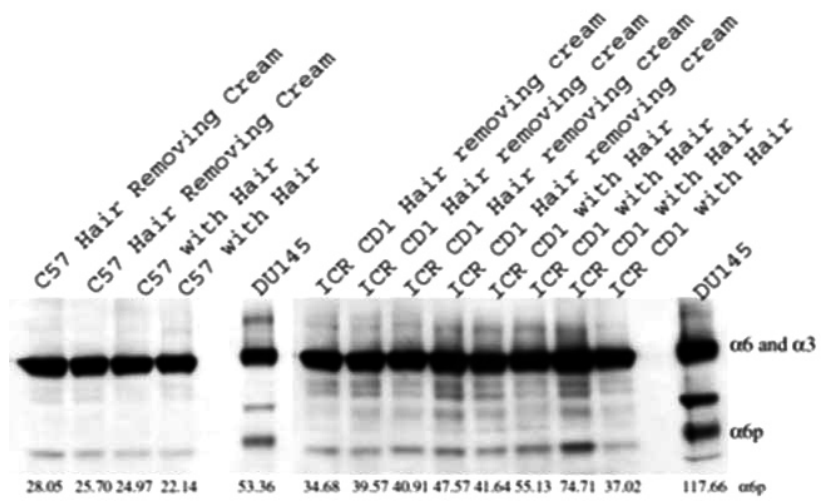

Fig. (1). Detectable expression of $\alpha 6 \mathrm{p}$ in normal mouse skin. Normal ICR and C57 mouse skin was analyzed for $\alpha 6$ or $\alpha 3$ and $\alpha 6 \mathrm{p}$ levels by western blotting. Two C57 and four ICR untreated mice were shaved using hair removing cream, and the whole skin was removed and frozen in liquid nitrogen. Another two C57 and four ICR untreated mice were left with the hair and the skin was then removed, and frozen in liquid nitrogen. The samples were processed and analyzed by western blotting. The $\alpha 6$ or $\alpha 3$ and $\alpha 6 \mathrm{p}$ integrin levels from DU145 cells are shown for comparative purposes. The relative numeric values of densitometic analysis are shown.

Since TPA and MNNG induce uPA expression in other systems, we utilized ICR mice in a tumor promotion model to analyze A6p levels in resulting tumors (Fig. 2). A single initiating dose of $100 \mu \mathrm{g}$ DMBA in acetone was applied topically to the shaved backs of female ICR mice. It was followed by bi-weekly application of $5 \mathrm{nM}$ TPA in acetone for 21 weeks. At week 22, TPA applications were stopped and replaced with weekly application of $120 \mu \mathrm{g}$ MNNG in acetone for a total of 40 weeks. The tumors were isolated and first analyzed by H\&E staining (data not shown) and classified by a pathologist as papillomas and squamous cell carcinomas (SCC). The tumors isolated from untreated control mice were analyzed by SDS-PAGE analysis for $\alpha 6$ or $\alpha 3$ and $\alpha 6 p$ integrin expression (Fig. 2). The results showed that $\alpha 6 \mathrm{p}$ was present in both papilloma and SCC tissues. Interestingly two of the papilloma tissue samples showed complete conversion of the $\alpha 6$ integrin to $\alpha 6 \mathrm{p}$. The presence of $\alpha 6 p$ without detecting the full length $\alpha 6$ or $\alpha 3$ has not been observed previously.

\section{$\alpha 6 p$ is Present in Melanomas of Transgenic Mice Ex-} pressing Activated Ha-Ras

In Fig. (3), we analyzed tumors isolated from transgenic mice that express an activated Ha-ras gene in their pig- 
mented cells that were described previously [19]. Melanoma tumors in different stages from these mice that were treated with DMBA were isolated and protein was extracted using RIPA lysis buffer. The results showed that all melanomas tested expressed high $\alpha 6 p$ levels.

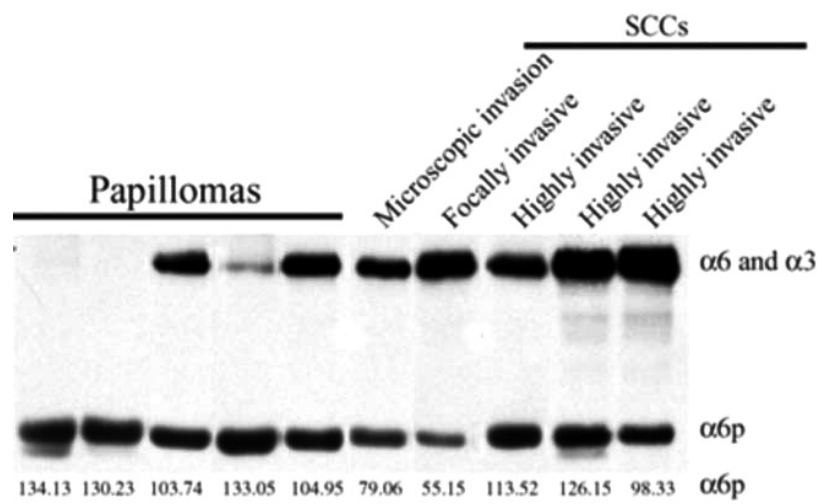

Fig. (2). $\alpha 6 p$ is present in mouse skin papillomas and squamous cell carcinomas. Papillomas and SCCs from ICR mice treated topically with a single initiating dose of DMBA, followed by twice weekly application TPA for 21 weeks and finally, at week 22, weekly application of MNNG for a total of 40 weeks, were collected and frozen in liquid nitrogen. The samples were homogenized by mortar and pestle, lysed in RIPA buffer and analyzed by western blotting. The relative numeric values of densitometic analysis are shown.

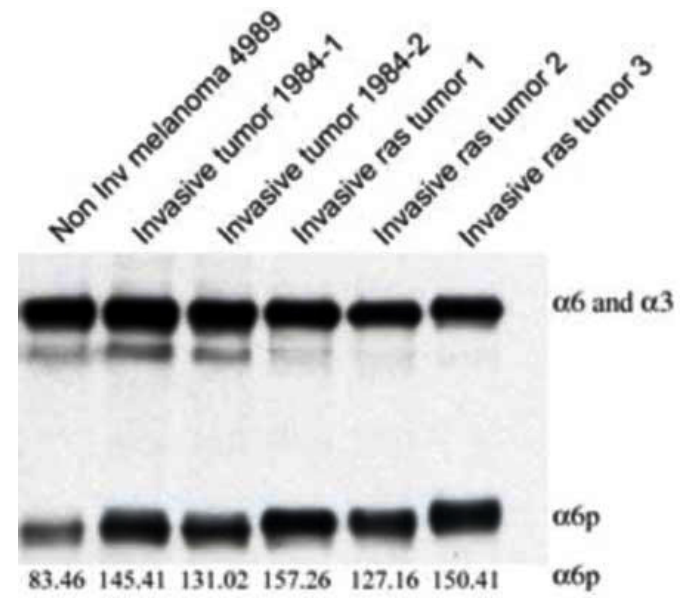

Fig. (3). $\alpha 6 p$ is present in melanomas from transgenic mice expressing an activated Ha-ras. Tumors in different stages were treated with DMBA, isolated and protein extracted using RIPA lysis buffer. The samples were then analyzed on an SDS-PAGE gel and the $\alpha 6 \mathrm{p}$ integrin was detected by western blotting. The relative numeric values of densitometic analysis are shown.

\section{Induction of $\alpha 6 \mathrm{p}$ In Vivo}

The next experiments were designed to determine whether cells growing as a tumor mass within an animal resulted in $\alpha 6 \mathrm{p}$ production. To accomplish this, we tested whether $\alpha 6 \mathrm{p}$ could be induced in tumors established by subcutaneous injection of a malignant cell line that does not contain endogenous $\alpha 6 \mathrm{p}$ under tissue culture conditions. The establishment of the malignant keratinocyte cell line (6M90) was described previously [16]. These cells were injected subcutaneously into athymic nude mice and the resulting tumors were isolated and protein was extracted using RIPA lysis buffer. In addition, RIPA lysis buffer was used to produce whole cell lysate from the 6M90 cell line. The samples were then analyzed by western blotting. The results showed that $\alpha 6 \mathrm{p}$ is present in all the tumors but absent in the cell line grown in tissue culture (Fig. 4).

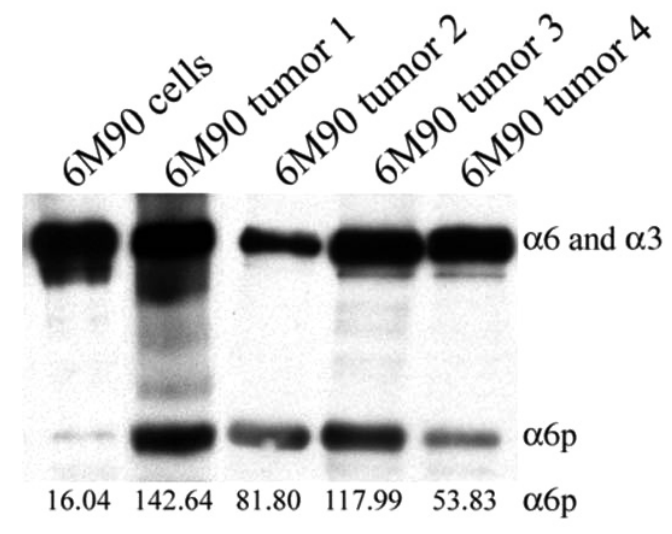

Fig. (4). The $\alpha 6 \mathrm{p}$ variant is induced in vivo. The establishment of the malignant keratinocyte cell line (6M90) was described previously [16]. The $6 \mathrm{M} 90$ cells were injected subcutaneously in athymic nude mice and the tumors were isolated and protein was extracted using RIPA lysis buffer. In addition, RIPA lysis buffer was used to produce whole cell lysate from the $6 \mathrm{M} 90$ cell line. The samples were then analyzed by western blotting. The relative numeric values of densitometic analysis are shown.

\section{DISCUSSION}

Our previous work has shown that the $\alpha 6 \mathrm{p}$ integrin is produced by uPA cleavage on the cell surface and that this cleavage is important in invasion and migration on laminin $[6,8]$. In addition, we have shown that $\alpha 6 \mathrm{p}$ is present in human prostate cancer tissue [6]. In this study we wanted to determine the $\alpha 6 \mathrm{p}$ integrin expression in normal mouse skin and skin tumor tissues. Several pieces of evidence indicate that UPA expression is induced after TPA treatments in different cell lines and on mouse skin [6, 13-15]. In addition, MNNG treatment has been shown to induce uPA expression in different cell lines $[15,20,21]$. Collectively, the above evidence led us to investigate the expression of $\alpha 6 \mathrm{p}$ in a mouse skin carcinogenesis model. Tumors from mice that were treated with DMBA, followed by TPA and then MNNG were analyzed for $\alpha 6 \mathrm{p}$ expression. The results showed that mice topically treated with DMBA, TPA followed by MNNG developed papillomas and squamous cell carcinomas that contained $\alpha 6 \mathrm{p}$ in high levels suggesting the involvement of $\alpha 6 \mathrm{p}$ during carcinogenesis. A very interesting finding was the fact that two of the papillomas showed complete conversion of the $\alpha 6$ integrin to $\alpha 6 \mathrm{p}$. This phenomenon has never been seen before in vivo or in vitro. $\mathrm{Pa}$ pillomas can progress, regress or remain stable. The observed variability in full length $\alpha 6$ and $\alpha 3$ expression in these samples could be due to a difference in the probability of progression. In addition, the results showed that $\alpha 6 \mathrm{p}$ is present at low but detectable levels in normal mouse skin and this suggests that $\alpha 6$ integrin cleavage may be involved in normal skin tissue remodeling. Collectively these data suggest that $\alpha 6 \mathrm{p}$ may be involved in normal skin tissue remodeling and during skin carcinogenesis. 
We then analyzed melanoma tumors from transgenic mice that express an activated Ha-ras gene in their pigmented cells. All the tumors analyzed contained high $\alpha 6 p$ levels. These data provide additional support to the emerging view that expression of $\alpha 6 p$ is not simply a tissue culture phenomenon and is found expressed in tumor types other than prostate cancer.

Our next objective was to determine whether the tumor microenvironment is important in the production of $\alpha 6 \mathrm{p}$ in vivo. We utilized a mouse keratinocyte cell line, 6M90, that does not express $\alpha 6 \mathrm{p}$ in vitro. Upon subcutaneous injection of the 6M90 cell line into athymic nude mice, the mice developed tumors. Analysis of these tumors by western blotting indicated that $\alpha 6 \mathrm{p}$ was induced. This suggests that growth of the tumor cells as a tumor mass will result in $\alpha 6 p$ production, perhaps through the stimulation of uPA activity within the tumor microenvironment.

Collectively, these data suggest that $\alpha 6 \mathrm{p}$ may be involved in both normal skin processes and during skin carcinogenesis. It is important to understand the function of $\alpha 6 p$ during these processes. Our studies so far with integrin $\alpha 6$ mutants, incapable of cleavage, have implicated $\alpha 6 \mathrm{p}$ in promoting tumor cell invasion and migration on laminin [8]. More recent work by others has proposed that $\alpha 6$ integrin is an adhesion molecule on progenitor or stem cells [23]. This raises an interesting possibility that cleavage of the ligand binding domain of $\alpha 6$ while preserving heterodimer formation may provide a mechanism of release within a stem cell like compartment. Testing of these ideas awaits further experimentation.

\section{ACKNOWLEDGEMENTS}

This work was supported by grants CA56666, CA40584, CA23074 and CA75152 from the National Cancer Institute and the Marie Curie IRG 036567.

\section{REFERENCES}

[1] Giancotti FG, Ruoslahti E. Integrin signaling. Science 1999; 285: 1028-32.

[2] Miranti CK, Brugge JS. Sensing the environment: a historical perspective on integrin signal transduction. Nat Cell Biol 2002; 4: E83-90.

[3] Schwartz MA, Schaller MD, Ginsberg MH. Integrins: emerging paradigms of signal transduction. Ann Rev Cell Deve Biol 1995; 11: 549-99.

[4] Green LJ, Mould AP, Humphries MJ. The integrin beta subunit. Int J Biochem Cell Biol 1998; 30: 179-84.

[5] Davis TL, Rabinovitz I, Futscher BW, et al. Identification of a novel structural variant of the alpha 6 integrin. J Biol Chem 2001; 276: $26099-106$.
[6] Demetriou MC, Pennington ME, Nagle RB, Cress AE. Extracellular alpha 6 integrin cleavage by urokinase-type plasminogen activator in human prostate cancer. Exp Cell Res 2004; 294: 550-8.

[7] Xiong JP, Stehle T, Zhang R, et al. Crystal structure of the extracellular segment of integrin alpha Vbeta3 in complex with an Arg-Gly-Asp ligand. Science 2002; 296: 151-5.

[8] Pawar SC, Demetriou MC, Nagle RB, Bowden GT, Cress AE. Integrin alpha6 cleavage: a novel modification to modulate cell migration. Exp Cell Res 2007; 313: 1080-9.

[9] Rickli EE. The activation mechanism of human plasminogen. Thromb Diath Haemorrh 1975; 34: 386-95.

[10] Gold LI, Schwimmer R, Quigley JP. Human plasma fibronectin as a substrate for human urokinase. Biochem J 1989; 262: 529-34.

[11] Naldini L, Tamagnone L, Vigna E, et al. Extracellular proteolytic cleavage by urokinase is required for activation of hepatocyte growth factor/scatter factor. EMBO J 1992; 11: 4825-33.

[12] Miyazawa K, Wang Y, Minoshima S, Shimizu N, Kitamura N. Structural organization and chromosomal localization of the human hepatocyte growth factor activator gene--phylogenetic and functional relationship with blood coagulation factor XII, urokinase, and tissue-type plasminogen activator. Eur J Biochem 1998; 258: $355-61$.

[13] Guerrini L, Casalino L, Corti A, Blasi F. NF-kappa B-mediated regulation of urokinase gene expression by PMA and TNF-alpha in human A549 cells. FEBS Lett 1996; 393: 69-73.

[14] Belin D, Godeau F, Vassalli JD. Tumor promoter PMA stimulates the synthesis and secretion of mouse pro-urokinase in MSVtransformed $3 \mathrm{~T} 3$ cells: this is mediated by an increase in urokinase mRNA content. EMBO J 1984; 3: 1901-6.

[15] Parra M, Lluis F, Miralles F, Caelles C, Munoz-Canoves P. The cJun N-terminal kinase (JNK) signaling pathway mediates induction of urokinase-type plasminogen activator (uPA) by the alkylating agent MNNG. Blood 2000; 96: 1415-24.

[16] Gupta A, Rosenberger SF, Bowden GT. Increased ROS levels contribute to elevated transcription factor and MAP kinase activities in malignantly progressed mouse keratinocyte cell lines. Carcinogenesis 1999; 20: 2063-73.

[17] Tamura RN, Rozzo C, Starr L, et al. Epithelial integrin alpha 6 beta 4: complete primary structure of alpha 6 and variant forms of beta 4. J Cell Biol 1990; 111: 1593-604.

[18] Cooper HM, Tamura RN, Quaranta V. The major laminin receptor of mouse embryonic stem cells is a novel isoform of the alpha 6 beta 1 integrin. J Cell Biol 1991; 115: 843-50.

[19] Powell MB, Hyman P, Bell OD, et al. Hyperpigmentation and melanocytic hyperplasia in transgenic mice expressing the human T24 Ha-ras gene regulated by a mouse tyrosinase promoter. Mol Carcinogenesis 1995; 12: 82-90.

[20] Brdar B, Matulic M. Induction of plasminogen activator by $\mathrm{N}$ methyl-N'-nitro-N-nitrosoguanidine in mer+ and mer- human tumour cell strains. Carcinogenesis 1988; 9: 2191-5.

[21] Brdar B. Induction of plasminogen activator by alkylating agents in a repair defective human glioblastoma cell strain. Cancer Res 1986; 46: $2282-4$.

[22] Demetriou MC, Cress AE. Integrin clipping: a novel adhesion switch? J Cell Biochem 2004; 91: 26-35.

[23] Xin L, Lukacs RU, Lawson DA, Cheng D, Witte ON. Self renewal and multilineage differentiation in vitro from murine prostate stem cells. Stem Cells 2007; 25: 2760-9. 